\title{
Abstraction and concretizing in information systems and problem domains: Implications for system descriptions
}

\author{
J.J.Kaasbøll \\ Department of Informatics, University of Oslo \\ P.O.Box 1080 Blindern, N-0316 Oslo, Norway, Phone: +47228524 10, \\ Fax: +4722852401, jens.kaasboll@ifi.uio.no, http://www.ifi.uio.no/ jensj
}

\begin{abstract}
"Abstraction" is used both for denoting relations in the problem domain of an information system, and for denoting relations inside software and hardware of a computer. The paper shows that separating abstraction in analysis of problem domains from abstraction when designing information systems resolves confusions concerning the direction of abstraction.

Abstraction is specialized in the paper as follows: representation, classification, generalization, aggregation, and role-realization. The latter relation occurs often when modelling reality, but it is presented with erroneous direction of abstraction in the literature.

Jackson System Development, object-oriented analysis and design, and dataflow diagrams can be improved through extensions with the abstraction relations specified in this paper.
\end{abstract}

\section{Keywords}

Modelling, analysis, design, object-orientation, system development methods, techniques

\section{INTRODUCTION}

Descriptions of information systems are often complex. One strategy of reducing complexity is to use abstraction. Abstraction should be supported by methods and learnt by students. Yet, "abstraction" is a diverse issue. In everyday language, abstraction is to ignore aspects of a phenomenon and concentrate on other aspects. Thus abstraction can be used to reduce complexity through ignoring the irrelevant aspects while focusing on the relevant ones.

Because of this need to reduce complexity, concepts and mechanisms for abstraction appear in programming languages, system description languages, user interface construction tools, and frameworks for information systems. "Information systems" here include programs, hardware, data, transformations and transportation of data, people interpreting and producing the data. The data, and the information that people have, refer to phenomena in problem domains.

Abstraction is useful both when analysing problem domains and when designing the software of an information system. However, abstraction for analysis and abstraction for design are seldom separated, which may lead to a confusion of the direction of abstraction and concretizing. 
A typical illustration of abstraction during analysis of the problem domain is found in (Olle et al, 1991). Olle et al state that "trading partner" is on a higher abstraction level than "supplier" and "customer" (p.58), and that users may comprehend a lower abstraction level easier than they understand a higher level.

Abstraction for design can be illustrated by a history of software abstraction as presented by Wirfs-Brock et al (1990, pp.4-5): the first abstraction was from bits to assembly language, then grouping instructions to macros and naming them, defining machine-independent high-level languages, grouping instructions into procedures, and defining abstract data types; the latter denotes a representation-independent specification of a data type that can be implemented in several ways. Abstraction in Wirfs-Brock et al's history deals with removing details of the computer from the software tools that are used for implementing application systems. Contrary to the analysis, users would probably understand a high level of abstraction in the design sense, eg, "customer," easier than a low level, eg, the ASCII code.

A low level of abstraction in analysis of problem domains is therefore not the same as a low level of abstraction during software design. This ambiguous meaning of "abstraction" illustrates the need for clarifying the concept, so that frameworks of information system concepts and techniques for development can distinguish and compare different types of abstractions without mixing them up.

Abstraction relations concerning the formal aspects of information systems have been discussed in the literature (Bergheim et al, 1989). This is useful for design and implementation of software, and it is in line with Wirfs-Brock et al's history. Abstraction relations of the problem domain have also been discussed (Haugen, 1980; van de Weg and Engmann, 1992). However, these do not relate abstractions in the problem domain and abstractions in the implementation of the information system, so that the ambiguity remains.

This paper aims to develop an unambiguous approach to abstraction that includes both analysis of problem domains and design of information systems. To achieve this, the "meaning triangle" constitutes the basis (Section 2). Two types of abstraction that relate the analysis and design approaches are discussed; representation (Section 3 and 4.1) and role-realization (Section 4.5 and 5.1). In addition to these concepts that are rarely considered in the information systems literature, the traditional relations of classification, generalization, and aggregation are briefly presented in Section 4. The abstraction relations are summarized in Section 4.6.

The corresponding view of information system (Section 5) and the resolution of the ambiguity of "abstraction" (Section 6) are presented. Implications of the approach on abstraction mechanisms in object- and flow-oriented techniques for system development is discussed (Section 7), and improvements are proposed.

\section{THE MEANING TRIANGLE}

In order to cover the problem domain, the information system, and the way the information system represents the problem domain, a traditional model of meaning and representation of reality (Holm and Karlgren, 1995) is chosen as the point of departure. In the model, an expression expresses a meaning, the meaning refers to an extension, and the expression represents the extension. These relations are depicted as a triangle, according to Charles Kay Ogden (1889-1957), see Figure 1.

The expressions consist of signs and symbols in the form of printed matter, sounds, contrasts on screens, electronic or magnetic patterns in computers. Extensions may consist of any phenomenon, also including expression and meaning. Hypothetical or future phenomena may 


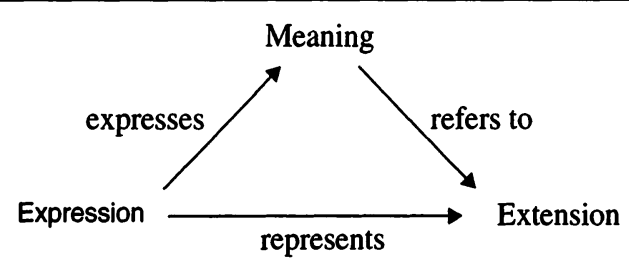

Figure 1: "Ogden's triangle": The relation between language and reality.

also be included in the extension. Meaning is the relation that persons make between expression and extension. Examples of meaning are the interpretation of an expression and the intention while carrying out a speech act.

When saying for short that an expression e represents a phenomenon $\mathrm{p}$ as its extension, it is assumed that the meaning that relates $e$ and $p$ is provided by relevant persons. These may be a language group if the expression is a word, or group of persons communicating if the expression is relevant for the group. We will therefore say for short that an expression represents a phenomenon, implicitly assuming the existence of appropriate persons who provides the relation through their meaning.

\section{REPRESENTATION}

An expression represents an extension if the meaning of the expression refers to the extension.

The relation between expression and extension is the representation relation, also for short representation.

When using the representation relation, extensions do not have to be present in a speech act, expressions are sufficient. If an expression cannot be separated in time and space from the phenomena which it represents, there is no representation relation.

Since abstraction is to ignore aspects and concentrate on those aspects that are relevant, and assuming that the expressions express the relevant aspects of the extension, representation is abstraction, because there are always aspects of a phenomenon that is not referred to by an expression.

Language is the most developed social means of representation. Since the relation between phenomena in the world and the expression depends on the linguistic conventions of the persons uttering or interpreting the expression, these persons are in the position of determining whether the relation holds.

Information systems process expressions through, eg, transforming and transporting data. The people working in an information system, and those whom the data concern, determine the meaning of the expressions in the system, and hence the extension represented by the expressions. When the expressions in the information system represent an extension, we will also say that the information system represents this extension. The extension of an information system is often called the "problem domain" or the "universe of discourse." Eg, parts of the expression at a flight ticket represent a flight journey when the persons issuing and controlling the ticket interpret it that way, see Figure 3. Correspondingly, the reservation system also represents the flight journeys, and the flight journeys constitute the problem domain (or the universe of discourse) of the system. The opposite of "to represent" is to be represented by. The 
journey is represented by the ticket.

Representations is a type of abstraction relations. The process starting with an extension, and associating an expression with it, is the representation process, which is an abstraction process, ie, a way of abstracting. The opposite of "an abstraction process" is a concretizing process, thus "to be represented by" is to concretize, eg, the journey concretizes the expression at the ticket.

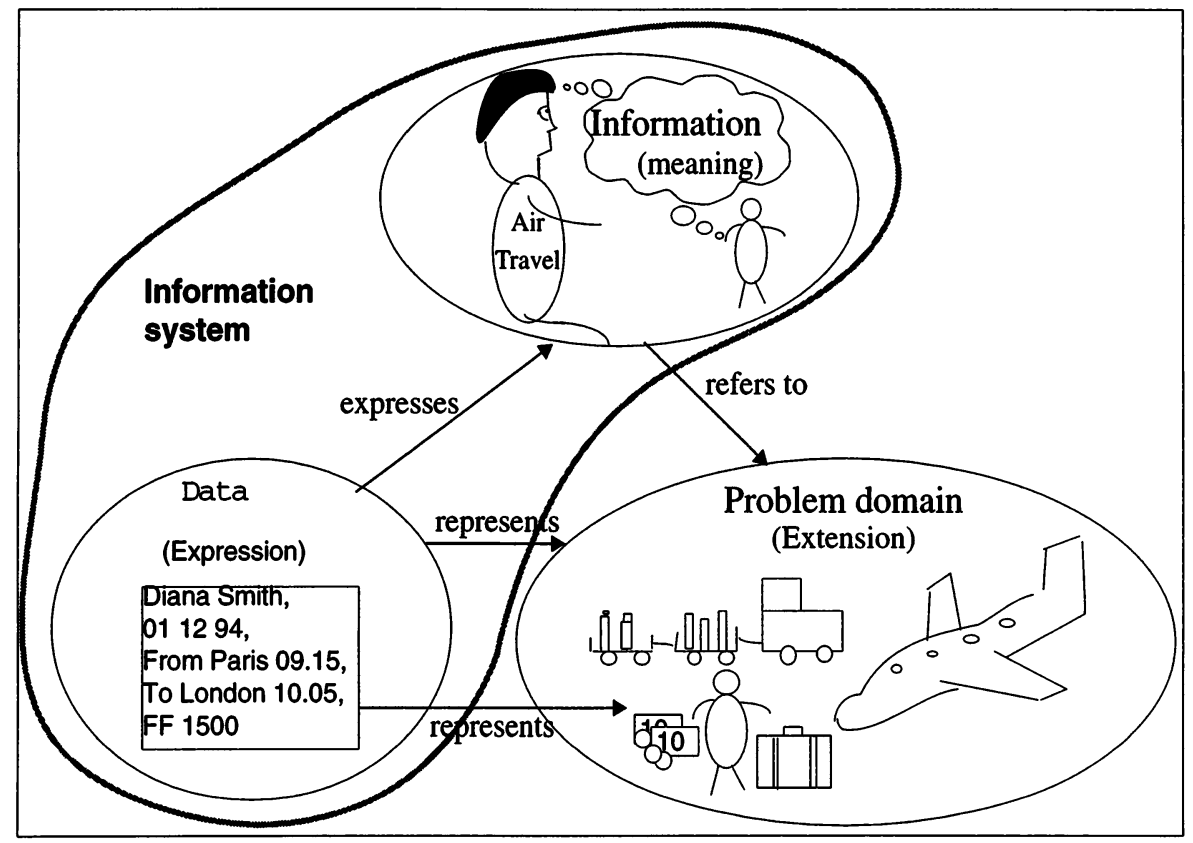

Figure 2: An information system consists of data that express the information of people in the system. The information system represents a problem domain.

\section{ABSTRACTION IN ANALYSIS OF PROBLEM DOMAINS}

\subsection{Representation}

Since extensions can be of any kind, there exist representation relations that have expressions or other representation relations as their extension. Assume that the pilots have the procedure knowledge written up as a text, which they follow during safety check of the airplane. Since the written procedure is an expression that represents each of the checks, there is a representation relation between the text and a check. Assume also that the computer system has the procedure in a knowledge base and, that this knowledge is used for control of the safety data. Then there is a representation relation inside the computer system that represents a corresponding representation relation in the problem domain.

In general, assume the following conditions:

- There exists a phenomenon $\mathrm{p}$

\begin{tabular}{|l|}
\hline \multicolumn{1}{|c|}{ Example } \\
\hline \hline safety check \\
\hline
\end{tabular}




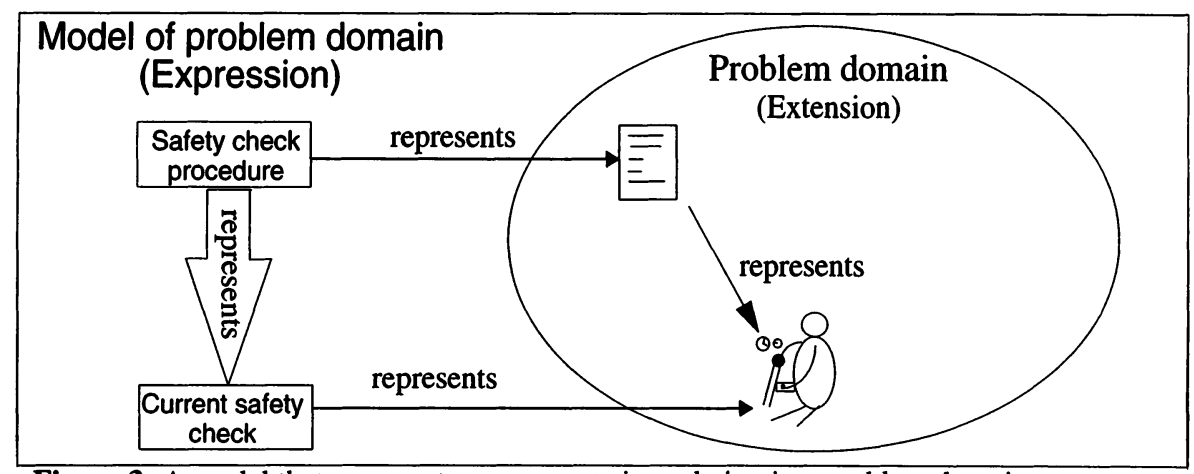

Figure 3: A model that represents a representation relation in a problem domain.

The meaning part of Ogden's triangle is omitted from the figure.

- There exists an expression pe in the problem domain, and pe represents $p$

- There exists an expression e in the information system, and e represents $p$

- There exists an expression ee in the information system, and ee represents pe

The procedure knowledge written up as a text represents safety checks

safety check data represents safety checks

the procedure in the knowledge base

represents the procedure knowledge

The representation relation "ee represents $e$ " represents the representation relation "pe represents $\mathrm{p}$. "

A criterion for the existence of a representation relation in the problem domain is that there exists an expression that can be separated in time and space from the phenomenon that the expression is about.

Knowledge based systems and expert systems represent general knowledge from the problem domain. The knowledge representations in the knowledge base is applied to specific data that represent phenomena in the problem domain.

\subsection{Classification}

This abstraction relation and the two following ones, generalization and aggregation, are assumed to be well known; thus they are not motivated and discussed. For motivation and discussion, consult textbooks on object-oriented analysis (Hutt, 1994) or articles on abstraction (eg, van de Weg and Engmann, 1992). The reason for presenting the relations here is to define them in the context of the meaning triangle.

For classification, assume the following conditions:

- There exists phenomena p1, Diana Smith's journey from Paris to London 01 12 94, Tom $\mathrm{p} 2, \ldots$

- There exist expressions e1 that represent $\mathrm{p} 1, \mathrm{e} 2$ that represents $\mathrm{p} 2, \ldots$

Jackson's journey from ..., ...

"Diana Smith, 0112 94, From Paris 09.15, To London 10.05, FF 1500 " represents Diana Smith's journey from Paris to London 0112 94; “Tom Jackson, 0112 94, From Paris To London 10.05, FF 1500" represents Tom Jackson's journey from ... 
- $e 1, e 2, \ldots$ have some common parts $\mathrm{E}$

- E represents a class $P$ consisting of $\mathrm{p} 1, \mathrm{p} 2, \ldots$

\begin{tabular}{|l|}
\hline “01 12 94, From Paris To London 10.05, FF 1500" \\
\hline $\begin{array}{l}\text { "01 12 94, From Paris To London 10.05, FF 1500" represents } \\
\text { journey from Paris to London } 011294 \ldots\end{array}$ \\
\hline
\end{tabular}

P classifies $\mathrm{p} 1, \mathrm{p} 2, \ldots$

Since only the common parts of $\mathrm{p} 1, \mathrm{p}, \ldots$ are included in $\mathrm{P}$, there are other parts that are left out, hence classification is abstraction. Since the parts of the expressions that are excluded in classification differ, these parts represent the differences between the phenomena of the extensions. Hence classification disregards differences while focusing on similarities.

The opposite of classification is instantiation, and $\mathrm{p} 1, \mathrm{p} 2, \ldots$ are instances or examples of the class $P$.

\subsection{Generalization}

Given two classes $\mathrm{C}$ and $\mathrm{B}, \mathrm{B}$ is a generalization of $\mathrm{C}$ if all instances of $\mathrm{C}$ are also instances of $B$.

Eg, "the right to travel" is more general than "the right to go by plane," because all instances of "the right to go by plane" also instantiate "the right to travel."

The opposite of generalization is specialization.

Since B may have instances that are not Cs, the common parts of the expressions of the Cs cannot be common for all B's. There has to be less common parts of the expressions of $C$ than those of $\mathrm{B}$. Thus some aspects of $\mathrm{C}$ are not aspects of $\mathrm{B}$, hence generalization is also abstraction.

\subsection{Aggregation}

Assuming the phenomena $\mathrm{p} 1, \mathrm{p} 2, \ldots$ are parts of $\mathrm{A}$,

$\mathrm{A}$ aggregates $\mathrm{p} 1$ and $\mathrm{p} 2$ and $\ldots$ into an aggregate. Conversely, $\mathrm{p} 1, \mathrm{p} 2, \ldots$ segregates A into details.

Eg, "Diana Smith, 01 12 94, From Paris 09.15, To London 10.05, FF 1500" and "Diana Smith, 011294 From London 11.15, To New York 13.00, FF 3000" aggregates to "Diana Smith, 01 12 94, From Paris 09.15, To New York 13.00, FF4500"

Aggregation differs from classification because the representations that are classified have to be similar in some respect, while similarity is no condition for aggregation.

\subsection{Role-realization}

Classification, generalization, and aggregation relate any phenomenon in the problem domain. The role-realization relation exists for those phenomena in the problem domain where at least two aspects can be identified. One aspect, the realization, is closer to physical matter than the other aspect, the role. For the role to exist, the realization also has to exist. Assume in general:

- There exists a phenomenon p-role in the problem domain
Diana Smith's actions and properties related to being a pilot. 
- Another phenomenon p-realization has also to exist for p-role to exist.

The person Diana Smith

Ro is a role of $\operatorname{Re}$. The opposite way: Re realizes $\mathrm{Ro}$.

The process of going from realization to role is called role-derivation, while the opposite direction is realization. Whereas separability in time and space is a criterion for identifying representation relations, roles exist in the same time and space as their realizations. Assuming that Diana Smith is also a passenger in the airline, both the pilot and the passenger are roles of the person. When Diana Smith disappears, so do both her roles too. Figure 4 illustrates the rolerealization relation.

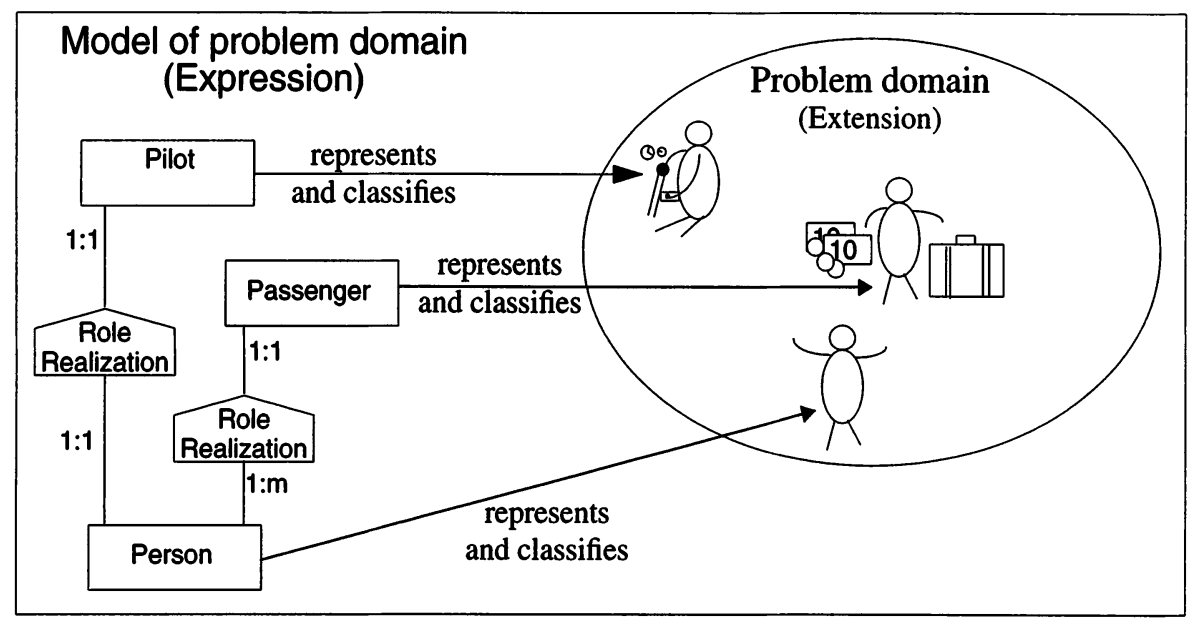

Figure 4: A model that represents two role-realization relations in a problem domain. All the three $\bigcup_{x} s$ in the problem domain are different aspects of the same Diana Smith.

In addition to persons realizing roles, phenomena that can be interpreted in symbolic ways also realize roles. For example, ink on paper realizes a text, a video cassette realizes a movie. For artificial realizations like a video cassette, there may exist many copies realizing the same role. One copy is necessary for the movie to exist. The necessity of the existence of the realization has not been considered in literature on role relations (Richardson and Schwarz, 1991; Coad, 1992; van de Weg and Engmann, 1992; Goldstein and Storey, 1994). In this paper, the existence criterion is important for deciding which aspect of a phenomenon is the role, and which is the realization. However, due to that the purpose here is to consider the direction of abstraction, the existence criterion will not be discussed further.

When separating roles from realizations, the aspects of the realization are ignored, hence roles are more abstract than their realizations.

An objection may be that the roles carry more information than the realizations, such that more aspects are considered when focusing on the roles rather than on the realizations. However, when the roles constitute the relevant issues, one can abstract away from the aspects of the realization, even if those are only a few. 
Counter to this, one can say that when the realization is in focus, the argument will work the other way around. However, since the realizations are closer to the physical world, more aspects of realizations can be found more easily than can aspects of roles.

A common way to make object-oriented models of roles, is to mix roles with specializations, see the example from (Odell, 1992) in Figure 5. Since the general class Person in Odell's model is more general than the classes Man, Woman, and Employee; Person is more abstract than Employee and other possible roles of a person. The same direction of abstraction is also suggested by Coad (1992) and van de Weg and Engmann (1992). This direction of abstraction is contrary to the direction that follows from the role-realization relation, as illustrated in Figure 6.

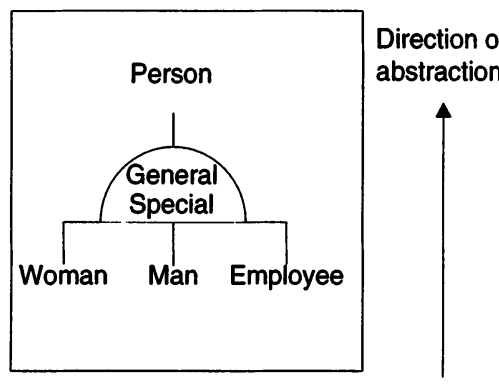

Figure 5: Mixing the role-realization relation with generalization according to Odell (1992)

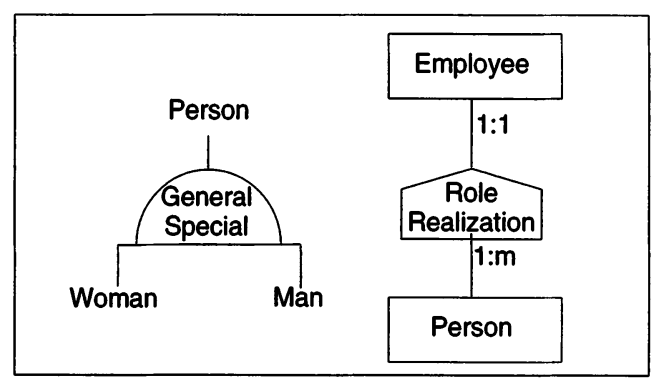

Figure 6: The role-realization relation and generalization with the proper direction of abstraction

Expressions consist of signs and other symbols, which is the subject of computer semiotics (Andersen, 1990). The form is the distinctive properties of the expression, while its substance is a particular way of realizing the form (Andersen, 1992, p.17). The form-substance relation is a specialization of the role-realization relation for expressions. When designing a text processor or other programs having expressions in their problem domain, several layers of form and substance may be needed.

\subsection{Relations between abstraction relations}

It has been argued that the representation, generalization, classification, aggregation, and rolederivation relations are abstractions. This means that every instance of these relations also instantiate "abstraction relation." Thus, these relations are specializations of "abstraction relation," and "abstraction relation" is a generalization of these relations, see Figure 7.

The corresponding concretizing relations are illustrated in Figure 8.

Classification and generalization are often regarded as unrelated mechanisms. As an exception, Tsichritzis and Lochovsky (1982) use the expression "token-type generalization" to denote classification (p.17). However, they neither argue for the relation, nor do they identify it as a generalization relation.

Specializations of aggregation have also been considered in literature (eg, van de Weg and Engmann, 1992). A transitive composition of whole from parts can be distinguished from a grouping of elements in a set (Motschnig-Pitrik, 1994). 


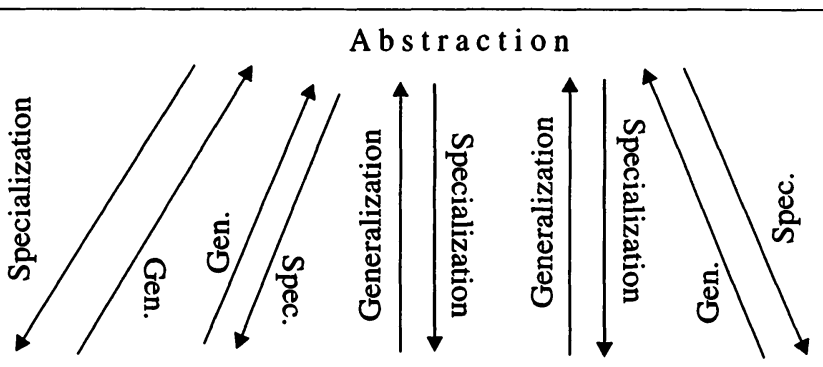

Representation Classification Generalization Aggregation Role-derivation

Figure 7: Abstraction relations: Generalization and specialization relations between abstraction relations.

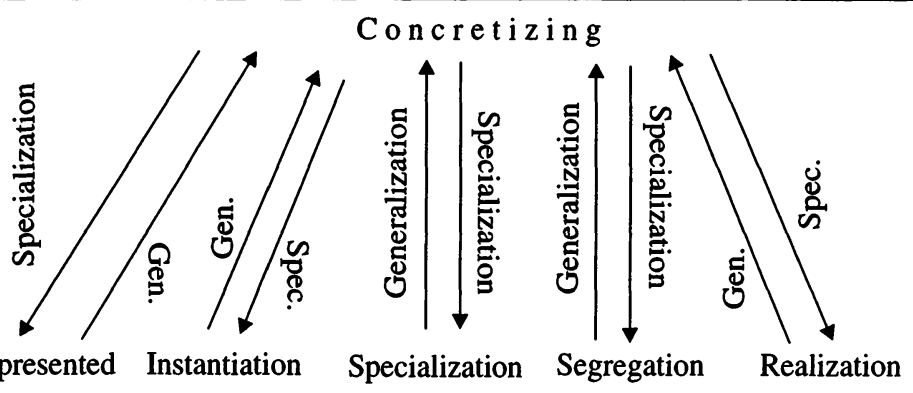

Figure 8: Concretizing relations.

\section{INFORMATION SYSTEM DESIGN}

\subsection{Role-realization relations}

Since information systems handle expressions, the role-realization relation is useful for structuring information systems. The expression "Diana Smith, 0112 94, From Paris 09.15, To London 10.05, FF 1500" can have a computer screen and a printed airline ticket as two different substances. The form is the shape that is invariant and recognized as the same meaning regardless of substance. Layers of realization can often be identified. Eg, black and white contrasts on computer screens can be substantiated with CRT and LCD substance.

Information systems do not only store data, they change data as well. The realization relation between form and substance can be extended to the transformation of data. Eg, when deleting a passenger from the database of scheduled flights, the selection of the passenger and of the text Delete that appears in a menu constitutes the form of the transformation. This form may be realized through, eg, pointing and clicking, or through pressing command keys. These realizations constitute substances.

Specifications are textual representations of program operation. During program execution, the operations are implemented in machine code and the data is also coded. Since the operations 
and data of a program execution expresses its meaning, the operations and data constitute its form, while the code is the substance of the program execution. Since it is common to talk about the textual representation of the program execution (the specification) instead of the execution, the relation is defined to be that the specification is realized in code. This special realization relation is called implementation, while the opposite is called specification-derivation, see Figure 9.

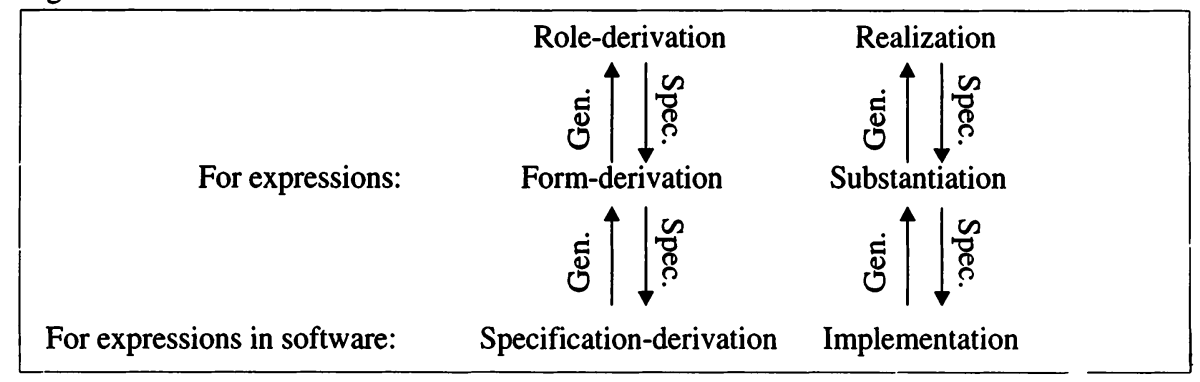

Figure 9: Role relations and realization relations in information systems.

Program execution is often organized in layers of implementation. Eg, text is implemented in ASCII code, which in turn is implemented in electrical currents and magnetic fields. These examples concern the computer systems as seen from a programmer's view. The above example of passenger and menu selection illustrates realizations of the program seen from a user's point of view.

Assume an implementation relation, known to be correct, between a specification and code. When the code is executed, it is highly predictable that the corresponding specification is followed, because errors tend not to appear at random.

The users also have to master realization relations. They have to know that to make the computer fulfil a specific function, specific behaviour is required. This is part of the skills required to master a system. However, people's knowledge and behaviour is not predictable to the same extent as computer processing. A user may push a button that triggers an unintended function. Therefore, there is a weaker regularity between the user's knowledge of the function and its implementation than between specification and implementation in the computer. Similarly, the relation between the form of a user's action and the substance of her/his behaviour is not as predictable as the realization relation of computers. Therefore, there exist other specializations of realization in addition to implementation.

To be able to deal with only one concept, the realization relation is defined in general for both computer processing and people's behaviour to be a regular correspondence between form and substance of signs, data, transformations of data, and categories of these. The collection of all code and the people, machines, documents, etc. that carry the code is called the ensemble of the information system. As in the theatre, the ensemble realizes the play. The realization relation during the operation of an information systems is illustrated in Figure 10.

\section{PROBLEM DOMAIN VERSUS INFORMATION SYSTEM}

Since realization in the information system does not interfere with abstraction and concretizing in the problem domain, the ambiguity of direction of abstraction presented in the introduction can be clarified. 


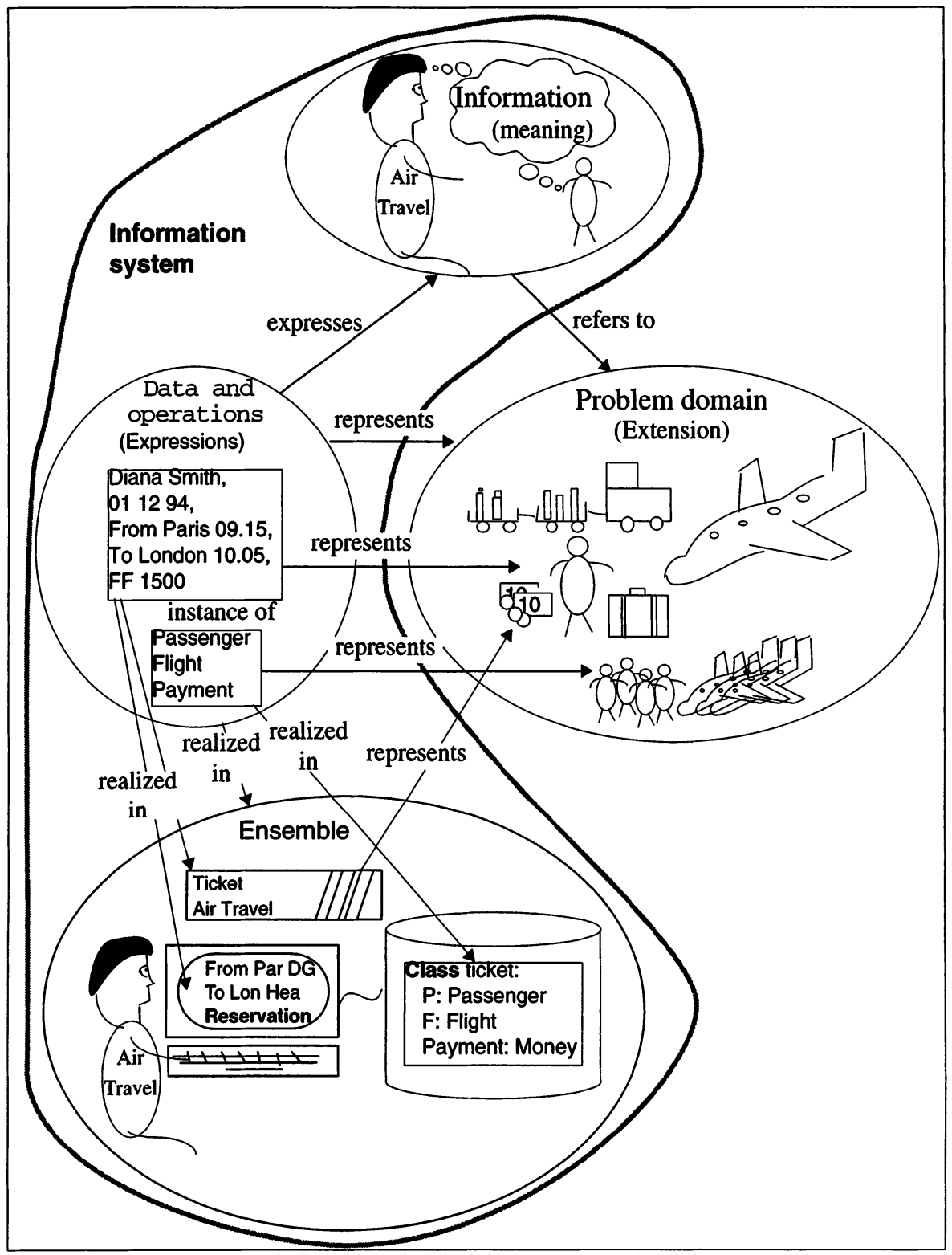

Figure 10: Representations and realizations during program execution in information systems. 
Confused direction of abstraction. Given an information system that represents a problem domain, eg, a flight reservation system. From the travel of Diana Smith from Paris to London, we can abstract "travels from Paris to London," from where we can abstract "travels." Then the question is, will it be more abstract or more concrete to go from "travels" (or from "Diana Smith, 0112 94, From Paris 09.15, To London 10.05, FF 1500") to program code, to $1011010100111001 \ldots$, and to electrical currents, light pulses, and magnetic fields? In the sense that abstraction is to ignore some aspects while focusing on others, electrical currents is certainly ignorance of everything concerned with flights, and focus on one issue. On the other side, electrical currents are more concrete than is a general concept like "travel." Thus there is intuitive support for saying both that realization in the information system is abstraction and concretization. Since both choices of direction of abstraction corresponds to some intuition, but runs counter to one another, separating abstraction in the problem domain from abstraction in the information system solves the confusion.

Confusion between users' and developers' perspectives. Since both users and developers strive for an accurate representation of the problem domain, they will have to deal with the same abstraction relations. When designing and implementing a solution, the developers will have to consider implementations in the information system. For periods of time, different layers of implementation may constitute the domain of the developers, making it reasonable to consider implementations in the computer as concrete, and specifications of applications as abstract. "Abstract data types" (Guttag and Horning, 1978) are examples. As long as the developers separate their technical sense of abstraction (specification-derivation and implementation) from abstractions in the problem domain, confusion is avoided. But if the developers mix up the two, they also mix their own technical view of an information system with the users' perspective.

The implementation relation is independent of the problem domain. Since implementation has no connection to the problem domain; the same implementations can be used for expressions representing any problem domain. Thus, by defining substantiation and implementation as relations between expressions only, the incoherence between the information system and the problem domain is taken care of.

Parts of the code may also represent. When analysing the information of a ticket, some of the printed text represents a travel. In addition, the material that the ticket is made of also represents phenomena in the problem domain. The specific paper and colours is a contract giving the passenger rights towards the airline company. The text at a photo copy of the ticket still represents the travel, while the copy is not representing the passengers rights. If developing a paperless computer based system for reservation and travel, the representation relations to the passengers rights must be taken care of. The functionality of the system is independent of the layer of realization where the expressions of the current system are found. Even if these representations were expressed by codes in the ensemble of the old system, it may be appropriate to make a textual expression in an electronic version of the system.

If the realization in the information system was mixed up with abstractions in the problem domain, those developing the system might have transferred the relation to the new system, knowing that the abstraction relations that corresponds to the problem domain should be transferred. 


\section{TECHNIQUES FOR ANALYSIS AND DESIGN}

Techniques for systems analysis and design are evaluated regarding their coverage of abstraction in the problem domain and the information system, and the evaluation is summarized in two tables. Three methods with distinct approaches are selected. Formal arguments are not provided, since the methods are assumed to be well-known. Suggestions for improvements are made.

\begin{tabular}{|l|l|l|l|}
\cline { 2 - 4 } \multicolumn{1}{c|}{} & \multicolumn{1}{c|}{ JSD } & \multicolumn{1}{c|}{ OOA/D } & \multicolumn{1}{c|}{ Dataflow } \\
\hline \hline Representation & No & No & No \\
\hline Classification & $\begin{array}{l}\text { The classification } \\
\text { process is supported, } \\
\text { but not the relation }\end{array}$ & $\begin{array}{l}\text { The classification } \\
\text { process is supported, } \\
\text { but not the relation }\end{array}$ & No. \\
\hline Generalization & $\begin{array}{l}\text { No. Could be } \\
\text { introduced }\end{array}$ & Yes & $\begin{array}{l}\text { No. Captured when } \\
\text { extended with data models }\end{array}$ \\
\hline Aggregation & $\begin{array}{l}\text { Of events only. Could } \\
\text { be extended }\end{array}$ & Yes & $\begin{array}{l}\text { No. Captured when } \\
\text { extended with data models. }\end{array}$ \\
\hline $\begin{array}{l}\text { Role- } \\
\text { Realization }\end{array}$ & No & No & No \\
\hline
\end{tabular}

\subsection{Jackson System Development}

Jackson System Development (JSD) was an early approach to modelling according to objects, called "entities" in the method (Jackson, 1983). JSD starts out with making a description of the problem domain. The method further advises to make a model of the data used to represent that domain and of the relations between the problem domain ("level 0") and the data ("level 1"). The descriptions may be extended to cover realizations of the data too ("levels 2 , ..."). JSD does not introduce any conceptual difference between representation and realization. The difference could probably easily be achieved if the two relations were introduced in the method, and "level 0" was called "problem domain"; "level 1" called "expression"; and "level 2 " and further levels called "layers of realization".

Classification of objects into classes is supported in the method, but there is no notation for describing a classification relation. However, it may be sufficient to say that all objects are treated as if they were instances of a class.

Unlike the object-oriented methods appearing around 1990, JSD does not support generalization. This could probably be remedied with a "gen/spec" relation similar to Coad and Yourdon's (1991a) technique. Additional rules for specifying how to merge event sequences when such sequences are inherited, would have to be provided. A proposal for such merges is found in (Mathiassen et al, 1992 and 1993).

The method has notation for aggregation of events, covering the common ways of structuring an algorithm. Aggregation of objects or classes is not supported. This could also be introduced, and the merging of event sequences could be carried out as for inheritance.

\subsection{Object-oriented analysis and design}

Coad and Yourdon's (1991a and b) method for object-oriented analysis and design (OOA/D) 
contain guidelines for analysis of the problem domain. In design, the description is to be interpreted as a model of the expressions in the computer. OOA/D therefore describes both the problem domain and the expressions that are going to represent it. However, because the method shift interpretation of the description instead of making separate descriptions, it becomes impossible to capture the representation relations between the problem domain and the expressions in the computer. Keeping the relations as in JSD could be done.

Similar to JSD, OOA advises classification, but the notation gives only room for the class, not for the relations between class and object. Generalization relations can be described through a "gen/spec" relation. Objects can be aggregated by means of a "whole-part" relation. In addition, objects can be aggregated in one level into "subjects."

During design, the model is implemented in user interface and in data base and task management implementations. Additional layers of implementation are not included. There seems to be no good reason why the method does not allow for any number of layers. The proposed realizations into user interface, data base, and task management may be practical solutions in many cases, but these could be specialized suggestions in a general mechanism for realizations.

\begin{tabular}{|l|l|l|l|}
\cline { 2 - 4 } \multicolumn{1}{c|}{} & \multicolumn{1}{c|}{ JSD } & \multicolumn{1}{c|}{ OOA/D } & \multicolumn{1}{c|}{ Dataflow } \\
\hline \hline $\begin{array}{l}\text { Representation } \\
\text { of problem } \\
\text { domain }\end{array}$ & $\begin{array}{l}\text { Yes. The problem domain } \\
\text { and relations to the data } \\
\text { that represents. Notation } \\
\text { can be improved. }\end{array}$ & $\begin{array}{l}\text { No. The problem } \\
\text { domain and the data } \\
\text { that represents are } \\
\text { described separately, } \\
\text { but the representation } \\
\text { relation is not captured. }\end{array}$ & $\begin{array}{l}\text { No. Can be achieved if } \\
\text { "material flow" and its } \\
\text { relation to "logical" } \\
\text { diagrams is included }\end{array}$ \\
\hline $\begin{array}{l}\text { Role- } \\
\text { realization }\end{array}$ & $\begin{array}{l}\text { Implementation can be } \\
\text { expressed in layers. Nota- } \\
\text { tion should be improved to } \\
\text { separate realization from } \\
\text { representation. }\end{array}$ & $\begin{array}{l}\text { Implementation in one } \\
\text { layer. Could be } \\
\text { extended to any } \\
\text { number of layers. }\end{array}$ & $\begin{array}{l}\text { Implementation in one } \\
\text { layer. Could be } \\
\text { extended to several } \\
\text { layers, provided a } \\
\text { suitable visualization. }\end{array}$ \\
\hline
\end{tabular}

\subsection{Structured analysis}

Structured analysis (DeMarco, 1978; Yourdon, 1989) consists of a series of techniques; here only dataflow diagrams are considered, since they constitute the core of the method. Dataflow diagrams describe "logical" and "physical" data processes, while "material processes" should be excluded in the dataflow technique. "Material processes" denotes what the data is about, being the problem domain. The representation relation is thus not included in dataflow diagrams. Through including material processes, the relation could be described, which is done in, eg, activity graphs in ISAC (Lundeberg et al, 1981). Dataflow diagrams are also extended with data dictionaries or data models. Extended ER models capture aggregation and generalization.

The "logical" diagrams describe the expressions in the system, while the "physical" diagrams depict the realization in one layer. However, the notation to show the relation between physical and logical is poor. The number of layers could be extended through introducing a "format" layer between the logical and the physical. A notation or visualization of the realization for each element in the layers is necessary for working with layers.

A process in a diagram can be expanded to show its interior, which may consist of more processes flows, and stores. The expansion is a mechanism for aggregation of the processes in 
the information system. However, aggregation in the interior of information systems is outside the scope of this paper.

\subsection{Summary of evaluation of techniques}

OOA/D supports generalization, aggregation, and classification in analysis of problem domains. JSD could be extended with similar mechanisms. Dataflow diagrams have poor support for analysis of problem domains. Their abilities to model aggregates of processes may be useful during design.

\section{CONCLUSION}

When abstraction during analysis of problem domains is mixed up with abstraction during design of information systems, the direction of abstraction becomes confused, and users' and developers' perspectives are mixed.

In addition to the well-known classification, generalization, and aggregation, two rarely considered abstractions have been discussed: representation and role-realization. Rolerealization often occur in problem domains. When defining the relation properly within the meaning triangle, it has been shown that models of roles and realizations found in literature erroneously indicated that realizations were more abstract than roles.

Techniques for modelling information systems can be improved through clarifying the different abstraction relations.

\section{Implications for practice}

System development techniques have been internalized by the developers using the techniques, and CASE tools may strengthen current practice and knowledge. Even if new methods that are more conceptually sound appear, those who are educated in accordance with the existing methods will probably stay within the modes of thought of these methods. It may be easier to change the CASE tools, so that they at least could allow for improvements of the methods and techniques. The CASE tools should be constructed to fulfil the following criteria:

- The tool represents the different abstraction relations both for the problem domain and the information system.

- The tool allows for defining notations that realize these relations as modifications and extensions of the techniques.

However, only changing computerized tools give little impact on knowledge and work practice (Sørensen, 1993). Improvements of system developers' knowledge about basic concepts like these relations should also be carried out.

\section{Further research}

Representation relations and role-realization relations in the problem domain should be studied through precise definitions, developing guidelines for modelling, empirical evaluations, and definitions of mechanisms in modelling techniques and programming languages.

\section{ACKNOWLEDGEMENT}

Thanks to Kristin Braa, Joan Greenbaum, Wolfgang Hesse, Renate Motschnig-Pitrik, Frieder Nake, Markku Nurminen, Antoni Olivé, Jan-Erik Ressem, Ole Smørdal, and the anonymous referees for discussions and comments on the paper, and to Sara Selmark for improving the language. 


\section{REFERENCES}

Andersen, P.B. (1990) A Theory of Computer Semiotics: Semiotic Approaches to Construction and Assessment of Computer Systems Cambridge University Press

Andersen, P.B. (1992) Computer Semiotics Scandinavian Journal of Information Systems Vol.4, pp.3-30

Bergheim, G.; Sandersen, E.; Sølvberg, A. (1989) A taxonomy of concepts for the science of information systems In Falkenberg, E. and Lindgreen, P. Information system concepts: an in-depth analysis ISCO 1, Amsterdam, North-Holland, pp.269-321

Coad, P. (1992) Object-Oriented Patterns Communications of the ACM 35, 9, pp.152-159

Coad, P. and Yourdon, E. (1991a) Object-Oriented Analysis Second Edition, Prentice-Hall, NJ

Coad, P. and Yourdon, E. (1991b) Object-Oriented Design Prentice-Hall, NJ

DeMarco, T. (1978)Structured Analysis and System Specification Yourdon, New York

Goldstein, R.C. and Storey, V.C. (1994) Materialization IEEE Transaction on Knowledge and Data Engineering $6,5, \mathrm{pp} .835-842$

Guttag, J.V. and Horning, J.J. (1978) The Algebraic Specification of Abstract Data Types Acta Informatica 10, pp.27-52

Haugen, Ø. (1980) Concepts of Hierarchies in Programming and System Description (In Norwegian) Master Thesis, Department of Informatics, University of Oslo

Holm, P. and Karlgren, K. Theories of meaning and different perspectives on information systems This volume

Hutt, A.T.F. (ed.) (1994) Object Analysis and Design: Description of Methods Wiley, New York

Jackson, M. (1983) System Development Prentice-Hall, New Jersey

Lundeberg, M.; Goldkuhl, G.; Nilsson, A. (1981) Information systems development: a systematic approach Englewood Cliffs, N.J.: Prentice-Hall

Mathiassen, L.; Munk-Madsen, A.; Nielsen, P. A.; and Stage, J. (1992) Modelling Events in Object-Oriented Analysis. In Bjerknes, Bratteteig \& Kautz (eds.) Precedings of the 15th IRIS Department of Informatics, University of Oslo, pp.742-757

Mathiassen, L.; Munk-Madsen, A.; Nielsen, P. A.; and Stage, J. (1993) Object Oriented Analysis (In Danish) Aalborg, Forlaget Marko

Motschnig-Pitrik, R. (1994) Analyzing the notions of attribute, aggregate, part, and member in data/knowledge modelling. In Zupancic and Wrycza (eds.) Proceedings of The Fourth International Conference Information Systems Development - ISD'94 Methods \& Tools. Theory \& Practice Moderna Organizacija, Kranj, pp.31-42

Odell, J. (1992) Managing object complexity, part I: abstraction and generalization Journal of Object-Oriented Programming 5, 5, pp.19-22

Olle, T. W.; Hagelstein, J.; Macdonald, I.G.; Rolland, C.; Sol, H.; Van Assche, F.J.M.; Verrijn-Stuart, A.A. (1991) Information Systems Methodologies: A framework for understanding 2nd Ed., Addison-Wesley, Wokingham

Richardson, J. and Schwarz, P. (1991) Aspects: Extending objects to support multiple, independent roles SIGMOD Record, Vol.20, No.2, pp.298-307

Sørensen, K. (1993) What influences regular CASE use in organizations? - An Empirically Based Model Scandinavian Journal of Information Systems 5, pp.25-50

Tsichritzis, D.C. and Lochovsky, F.H. (1982) Data models Prentice-Hall, Englewood Cliffs, NJ

van de Weg, R. L.W. and Engmann, R. (1992) A framework and Method for Object-Oriented Information Systems Analysis and Design. In E.D. Falkenberg, C. Rolland, and E.N. El-Sayed (eds.) Information System Concepts: Improving the Understanding ISCO 2, IFIP Transactions A-4, North-Holland, pp.123-146

Wirfs-Brock, R.; Wilkerson, B.; Wiener, L.(1990) Designing Object-Oriented Software Prentice-Hall, NJ

Yourdon, E. (1989) Modern Structured Analysis Yourdon Press, Englewood Cliffs, NJ

\section{BIOGRAPHY}

Jens Kaasbøll is assistant professor at the Department of Informatics, University of Oslo. He has published in Information Systems Journal, in Journal of Object-Oriented Programming and in conferences. He has served as a co-editor of Scandinavian Journal of Information Systems. His research interests are both in the theoretical basis for information systems, including objectoriented modelling, and in finding practical ways to redesign systems so that they are better suited to their tasks and appear more integrated seen from users' points of view. 